\title{
Application of Digital Tomosynthesis in the Diagnosis of Urolithiasis: Comparison with MDCT
}

\author{
Shifeng Liu, MD, ${ }^{1}$ Pei Nie, MD, ${ }^{2}$ Hong Wang, MS, ${ }^{3}$ Jian Guo, MS, ${ }^{2}$ Qingjun Shang, MS, ${ }^{4}$ \\ Wenjian $\mathrm{Xu}, \mathrm{PhD}^{2}$ and Weihua Feng, $\mathrm{PhD}^{2}$
}

\begin{abstract}
Objective: To investigate the diagnostic value of digital tomosynthesis in urolithiasis compared with multidetector computed tomography (MDCT).

Subjects and Methods: This study received the approval of institutional review board and obtained the informed consents of all subjects. Fifty patients with acute renal colic and hematuria from February 2014 to February 2015 were enrolled. They underwent both MDCT and digital tomosynthesis. Images were interpreted by two experienced radiologists, and inter-reader agreement was assessed. The diagnostic performance of urinary stones with digital tomosynthesis and MDCT was compared. The entrance surface dose (ESD) in MDCT and digital tomosynthesis for patients was measured and compared.

Results: No significant difference was found between digital tomosynthesis and MDCT in the diagnosis of urinary stones $\left(X^{2}=1.357, p>0.05\right)$. The ESD in digital tomosynthesis was lower than that in both routine-dose and low-dose MDCT for patients $(p<0.05)$.

Conclusions: Digital tomosynthesis has great diagnostic value in urolithiasis. Compared with MDCT, digital tomosynthesis can significantly decrease ESD for patients and financial loan with a similar detection rate.
\end{abstract}

Keywords: digital tomosynthesis, MDCT, urolithiasis, entrance surface dose

\section{Introduction}

$\mathbf{U}$ RINARY TRACT STONES DISEASE frequently influences younger patients, and the incidence increases year by year with a high risk of recurrence. ${ }^{1}$ Therefore, effective and accurate diagnosis of urinary stones is of great value in treatment and prognosis. Many imaging modalities have been applied to diagnose urinary stones, such as the radiography of kidney, ureter, and bladder (KUB), the ultrasonography (US), the intravenous pyelography (IVP), and the CT.

Radiography of KUB has been well recognized as the commonly used approach in diagnosing urinary stones. Although the radiography has a good positioning capability, its sensitivity to urinary stones is low (58\%-62\%) because of its susceptibility to intestinal gas and bones and its low spatial resolution. ${ }^{2-4}$ US is suggested as an effective imaging modality in screening acute renal obstruction caused by nephrolithiasis for its low cost and ready availability. ${ }^{5,6}$ Nevertheless, the sensitivity of US to renal stones is only $24 \%$ to $60 \%{ }^{7,8}$ Published literature ${ }^{9}$ showed that IVP has a higher sensitivity and specificity in diagnosing urinary stones, even up to $90 \%$, but intravenous use of contrast agent may increase its side effects such as allergy, and the relatively high dose of ionizing radiation limits its wide application. ${ }^{10,11}$ Unenhanced multidetector computed tomography (MDCT) has been extensively regarded as the most sensitive imaging tool in diagnosing urinary stones, and its sensitivity is as high as $97 \%$ according to previous reports. ${ }^{12-17}$ Because of its high space resolution and twodimensional (2D) and the three-dimensional (3D) postprocessing images, MDCT can better display the location, shape, and size of the stones and show the contour of kidneys, ureters, and the bladders. However, the application of MDCT in diagnosis or follow-up has attracted attention to the radiation exposure and the financial burden. Although the proposed low-dose MDCT regimen reduced the radiation dose to some extent, MDCT is still not considered the preferred method in the diagnosis and follow-up of urinary stones. ${ }^{18-21}$

Digital tomosynthesis is a new method relying on many 2D radiographs of interesting object acquired at various angles. A coronal image plane may then be reconstructed from the 2D

\footnotetext{
${ }^{1}$ Interventional Medical Center; ${ }^{2}$ Department of Radiology, The Affiliated Hospital of Qingdao University, Qingdao, China.

${ }^{3}$ Dermatology Department, Qing Dao No. 6 People's Hospital, Qingdao, China.

${ }^{4}$ Department of Tumor Radiotherapy, The Affiliated Hospital of Qingdao University, Qingdao, China.
} 
Table 1. Size and Location Dependency of Sensitivity

\begin{tabular}{|c|c|c|c|c|c|c|c|c|c|}
\hline \multirow[b]{2}{*}{ Examinations } & \multicolumn{4}{|c|}{$<5 \mathrm{~mm}$} & \multicolumn{4}{|c|}{$>5 \mathrm{~mm}$} & \multirow[b]{2}{*}{ Total } \\
\hline & Kidney & Ureter & Bladder & Total & Kidney & Ureter & Bladder & Total & \\
\hline Digital tomosynthesis & 13 & 20 & 0 & 33 & 23 & 30 & 0 & 53 & 86 \\
\hline MDCT & 13 & 20 & 0 & 33 & 23 & 30 & 0 & 53 & 86 \\
\hline MDCT-2D-Mip & 11 & 20 & 0 & 31 & 22 & 30 & 0 & 52 & 83 \\
\hline
\end{tabular}

2D-MIP = two-dimensional-maximum intensity projection; $\mathrm{MDCT}=$ multidetector computed tomography.

data by spatially translating each $2 \mathrm{D}$ image and superimposing each radiograph to an arbitrary reference. The advantage of this technique is that it can remove overlapping structures and provide in-depth information, so more tiny and occult structures can be displayed. ${ }^{22}$ Digital tomosynthesis has already been used to evaluate breast, airway, lung, hand, and dental disorders in clinic, ${ }^{22-27}$ but there are few reports on its application to urinary diseases. The current research was intended to compare the performance and radiation dose of digital tomosynthesis and MDCT in diagnosing urolithiasis.

\section{Subjects and Methods}

Patients

This study was approved by the institutional review board of the Affiliated Hospital of Qingdao University (No. 33687). All participants were informed of the purpose of this study and signed the written informed consent.

Fifty consecutive patients ( 34 men and 16 women) whose age ranged from 22 to 64 years (mean age: 38 years; median age: 40 years) with acute renal colic and hematuria from February 2014 to February 2015 were enrolled in this prospective study. The inclusion criterion was that the patients with renal colic showed urinary stones on CT, and the exclusion criterion was that the patients with renal colic did not show urinary stones on CT. All patients underwent emergent MDCT examination to determine the diagnosis of urinary stones. After the acute renal colic was relieved, these patients underwent additional digital tomosynthesis.

\section{Multidetector computed tomography}

A 16-MDCT scanner (Light Speed, GE Healthcare) was employed to conduct MDCT according to the protocols of routine-dose and low-dose scanning. Both the MDCT scanning were performed in a helical mode. For MDCT at a routine dose, the tube voltage and the tube current time of scanning were $120 \mathrm{kVp}$ and $150 \mathrm{mAs}$, respectively. For MDCT at a low dose, the tube voltage and the tube current time of scanning were $100 \mathrm{kVp}$ and $100 \mathrm{mAs}$, respectively. The pitch of the two MDCT scanning was 0.8 , and the section thickness was $1.25 \mathrm{~mm}$.
The tissue algorithm was utilized to reconstruct the images with a section thickness of $1.0 \mathrm{~mm}$ and an interval of $0.7 \mathrm{~mm}$. Coronal two-dimensional-maximum intensity projection (2D-MIP) was reconstructed for image interpretation.

\section{Digital tomosynthesis}

Digital tomosynthesis was performed with a multipurpose digital tomosynthesis machine (Shimadzu X-ray digital flatpanel fluoroscopy/camera system). For digital tomosynthesis of the abdomen, a rotation angle of $40^{\circ}$ was used, and the distance between $\mathrm{x}$-tube and the check bed was set as $1100 \mathrm{~mm}$. Patients were placed in supine position and scanned in an anteroposterior view at 90 to $110 \mathrm{kVp}, 4$ to $6 \mathrm{mAs}$. All the images were additionally reconstructed in the coronal view at height, 130 to $150 \mathrm{~mm}$; range, $100 \mathrm{~mm}$; slice thickness, $2 \mathrm{~mm}$.

\section{Image analysis}

All the images were transferred to the PACS/HIS (picture archiving and communication systems and hospital information system) and were independently read by two experienced radiologists ( 8 and 11 years of abdominal imaging experience). The two radiologists were uninformed about the $\mathrm{CT}$ results and were informed that all the patients had acute renal colic and hematuria. All the stones presented on the axial and coronal 2D-MIP CT images and digital tomosynthesis were recorded. Images could display the presence, number, and location of the stones. Agreement was reached on any disagreement between the two readers in their assessment.

\section{Entrance surface dose measurement}

Among the 50 patients, 15 of them were randomly selected to evaluate the entrance surface dose (ESD). The ESD in routine-dose and low-dose MDCT and digital tomosynthesis for patients were measured with the same protocol as the 15 selected patients using the dose 1 dosimeter (IBA Company). Its finger-like lionization chamber detector (FC65-G) was placed in the same position as the stones found in the 15 patients. The error of the measurement value, which was $<1 \%$, was satisfied with the international standard $(<2 \%)$.

Table 2. Examination Dependency of Sensitivity Comparison

\begin{tabular}{|c|c|c|c|c|c|c|}
\hline \multirow[b]{2}{*}{ Examinations comparison } & \multicolumn{2}{|c|}{ Total stones } & \multicolumn{2}{|c|}{$<5 \mathrm{~mm}$} & \multicolumn{2}{|c|}{$>5 \mathrm{~mm}$} \\
\hline & $X^{2}$ & $\mathrm{p}$ & $X^{2}$ & $\mathrm{p}$ & $X^{2}$ & $\mathrm{p}$ \\
\hline Digital tomosynthesis $v s$ MDCT & - & - & - & - & - & - \\
\hline Digital tomosynthesis $v s$ MDCT-2D-Mip & 1.357 & 0.246 & 0.516 & 0.492 & 0.000 & 1.000 \\
\hline MDCT vs MDCT-2D-Mip & 1.357 & 0.246 & 0.516 & 0.492 & 0.000 & 1.000 \\
\hline
\end{tabular}



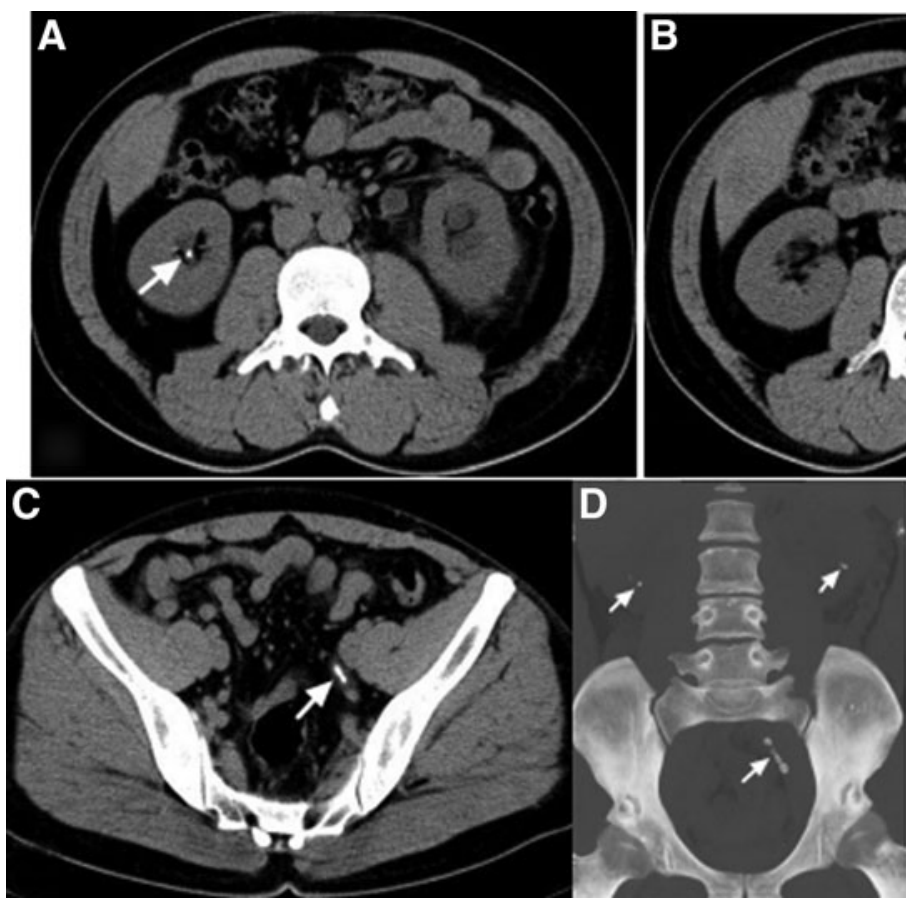
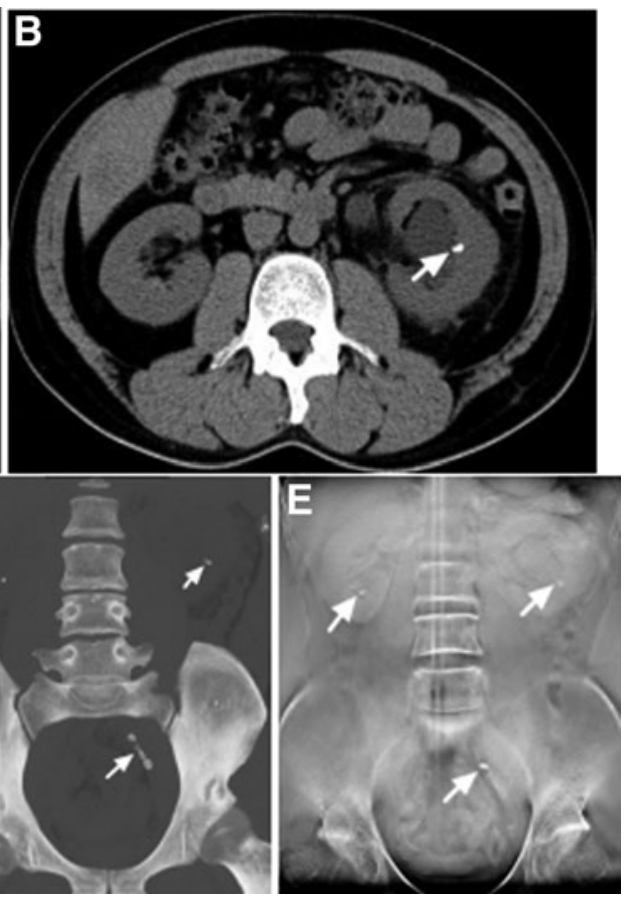

FIG. 1. A 36-year-old man with urinary stones. Axial MDCT images (A-C) show numerous stones in both kidneys and the left ureter. Coronal 2D-MIP CT (D) and coronal digital tomosynthesis (E) images have the same imaging manifestation as the axial MDCT image. They all can show the calculi and the contour of two kidneys clearly. 2D-MIP $=$ twodimensional-maximum intensity projection; $\mathrm{MDCT}=$ multidetector computed tomography.

\section{Cost-effectiveness analysis}

The rate of cost effectiveness was calculated according to the following formula: cost-effectiveness rate $(\mathrm{RMB} /$ stone $)=$ the total cost/the number of stones detected. Cost-effectiveness analysis was used to explore the most economical way in detecting urinary stones by comparing the relative costs and outcomes (effects) of the four groups.

\section{Statistics analysis}

All the data were calculated with statistical software (SPSS). With the multireader and multicase data sets, McNemar test was used to evaluate the difference among MDCT-2D-MIP, routine-dose MDCT, and digital tomosynthesis regarding detection rate. The difference of the ESDs among low-dose MDCT, routine-dose MDCT, and digital tomosynthesis groups

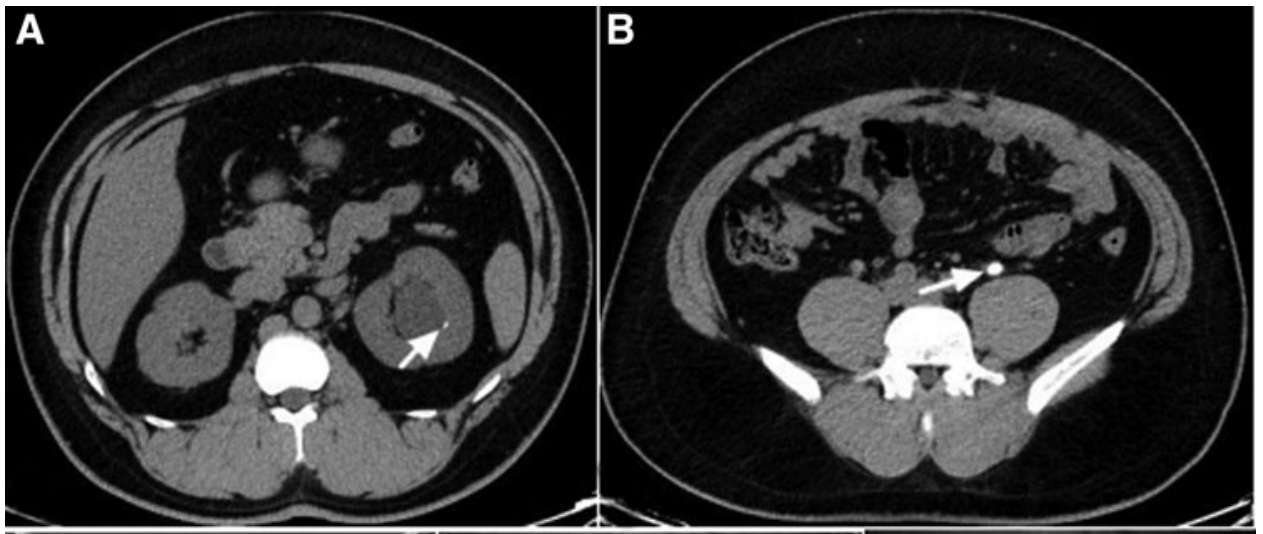

FIG. 2. A 30-year-old man with urinary stones. Axial MDCT images $(\mathbf{A}, \mathbf{B})$ show one stone in the left kidney and one stone in the left ureter. Coronal 2D-MIP CT (C) and coronal digital to-

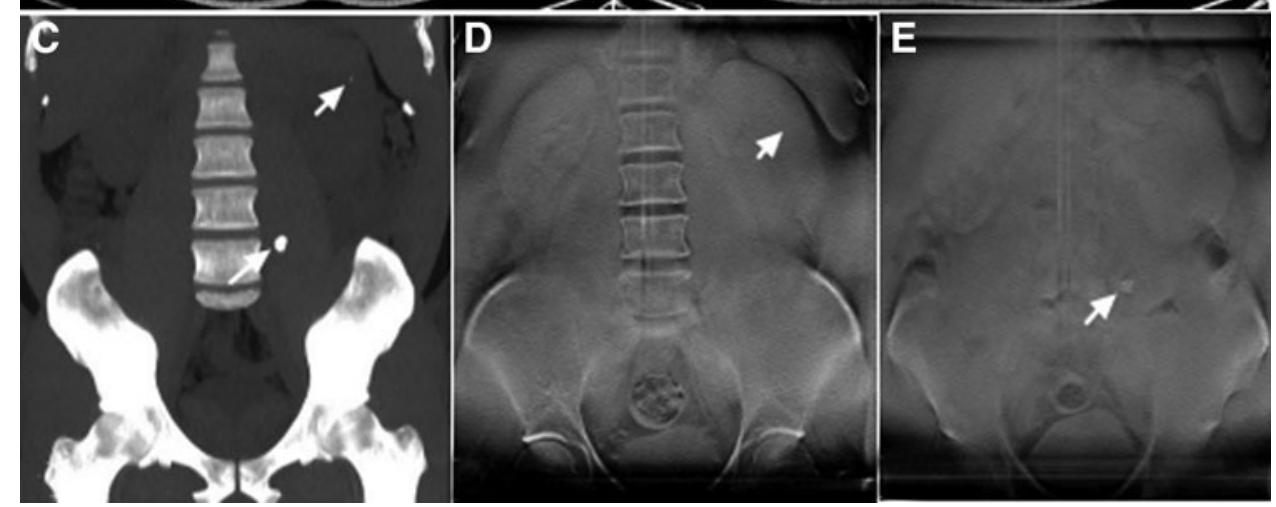
mosynthesis (D, E) images have the same imaging manifestation as axial MDCT image. They all can show the calculi and the contour of two kidneys clearly. 
Table 3. Mean Radiation Dose Measured With ANTHROPOMORPHIC Phantom ON Digital TOMOSYNTHESIS, Low-Dose MDCT, AND RouTINE-Dose MDCT

\begin{tabular}{cccc}
\hline Patients & $\begin{array}{c}\text { Digital } \\
\text { tomosynthesis } \\
\left(\text { Gycm }^{2}\right)\end{array}$ & $\begin{array}{c}\text { Low-dose } \\
\text { MDCT } \\
\left(G y \mathrm{~cm}^{2}\right)\end{array}$ & $\begin{array}{c}\text { Routine } \\
\text { MDCT } \\
\left(G y \mathrm{~cm}^{2}\right)\end{array}$ \\
\hline 1 & 8.47 & 13.16 & 50.96 \\
2 & 8.63 & 13.18 & 51.03 \\
3 & 8.09 & 13.13 & 50.88 \\
4 & 6.86 & 13.06 & 50.68 \\
5 & 9.76 & 13.33 & 51.26 \\
6 & 9.24 & 13.27 & 51.15 \\
7 & 8.94 & 13.23 & 51.08 \\
8 & 7.58 & 13.1 & 50.82 \\
9 & 8.63 & 13.18 & 51.03 \\
10 & 8.63 & 13.18 & 51.03 \\
11 & 8.09 & 13.13 & 50.88 \\
12 & 10.48 & 13.39 & 51.31 \\
13 & 9.24 & 13.27 & 51.15 \\
14 & 8.47 & 13.16 & 50.96 \\
15 & 8.94 & 13.23 & 51.08 \\
$\bar{X}$ & 8.67 & 13.2 & 51.02 \\
\hline
\end{tabular}

was analyzed by the paired-sample $t$-test. The interobserver variability of digital tomosynthesis and CT in detecting urinary stones was assessed with a $k$ statistic $(\kappa>0.81$, excellent agreement; $\kappa=00.61$ to 0.80 , good agreement). $p$ value $<0.05$ indicated statistically significant.

\section{Results}

Stones have been detected in all 50 patients. Emergent CT (axial and coronal 2D-MIP images) showed a total 86 urinary stones ( 33 stones $<5 \mathrm{~mm}, 53$ stones $>5 \mathrm{~mm}$ ) in 50 patients with acute renal colic; 36 stones were observed in the kidney ( 13 stones $<5 \mathrm{~mm}, 23$ stones $>5 \mathrm{~mm}$ ) and the others were found in the ureter ( 20 stones $<5 \mathrm{~mm}, 30$ stones $>5 \mathrm{~mm}$ ). No bladder stones were discovered (Table 1). Digital tomosynthesis presented 83 urinary stones, and the sensitivity was $94 \%$ (83/86). Both the positive and the negative predictive rates of MDCT and digital tomosynthesis were $100 \%$. The results of $\mathrm{McNe}-$ mar test showed that the performance of digital tomosynthesis was as good as that of MDCT in detecting the presence (ignoring the size and location) of urinary stones (Table 2).

Table 4. Cost-EfFectiveness Rates OF DIFFERENT EXAMINATIONS

\begin{tabular}{lccc}
\hline & $\begin{array}{c}\text { Total } \\
\text { cost } \\
(\text { RMB })\end{array}$ & $\begin{array}{c}\text { Effectiveness } \\
(E, \%)\end{array}$ & $\begin{array}{c}\text { Cost-effectiveness } \\
\text { rate }(\text { RMB/stone })\end{array}$ \\
\hline $\begin{array}{l}\text { Digital } \\
\text { tomosynthesis }\end{array}$ & 4000 & 96.51 & 41.45 \\
$\begin{array}{l}\text { Low-dose } \\
\text { MDCT }\end{array}$ & 20000 & 100.00 & 200.00 \\
$\begin{array}{l}\text { Routine-dose } \\
\text { MDCT }\end{array}$ & 20000 & 100.00 & 200.00 \\
$\begin{array}{l}\text { MDCT-2D-Mip } \\
\text { MDCT-25000 }\end{array}$ & 100.00 & 250.00 \\
\hline
\end{tabular}

Cost-effectiveness rate $=$ total $\cos / /$ the number of stones.
What's more, both digital tomosynthesis and MDCT could clearly display the stones and contour of the kidneys (Figs. 1 and 2).

The results of the two diagnostic methods of MDCT and digital tomography were consistent between observers, with $k$ values of 0.85 and 0.82 , respectively.

Good agreement between readers was found in diagnosing urinary stones both by MDCT and by digital tomosynthesis method, and the corresponding $k$ values were 0.85 and 0.82 , respectively. When the data were analyzed at the patient level (whether there were stones in a patient) and at the stone level (whether there were stones in a particular site), the findings were accordant.

The mean ESD of abdominal digital tomosynthesis was $8.67 \mathrm{Gycm}^{2}$, which was much lower than that in both routine-dose MDCT (51.02 $\left.\mathrm{Gycm}^{2}\right)$ and low-dose MDCT $\left(13.20 \mathrm{Gycm}^{2}\right)$. The paired-sample $t$-test revealed that the $p$ value of comparison between two groups was $<0.05$ (Table 3).

Cost-effectiveness rates obtained from four examinations are shown in Table 4. The total cost of digital tomosynthesis group was $4000 \mathrm{RMB}$, which was significantly lower than that of low-dose MDCT group (20,000 RMB), routine-dose MDCT group (20,000 RMB), and MDCT-2D-Mip group $(25,000$ $\mathrm{RMB})$. The cost-effectiveness rate of digital tomosynthesis was 41.45 RMB/stone. Low-dose MDCT and routine-dose MDCT both had a much higher cost-effectiveness rate of 200.00 $\mathrm{RMB} /$ stone. There was a significant difference in the costeffectiveness rate between digital tomosynthesis and low-dose MDCT group $(p<0.05)$. MDCT-2D-Mip had the highest costeffectiveness rate of $250.00 \mathrm{RMB} /$ stone among the four examinations.

\section{Discussion}

This study demonstrated that digital tomosynthesis is as repeatable between observers as MDCT. Interobserver agreement was almost as good with digital tomosynthesis as with MDCT.

Our study also showed that when measured with anthropomorphic phantom, routine-dose MDCT had the highest ESD, while the ESD in digital tomosynthesis was the lowest. A previous study ${ }^{28,29}$ showed that the radiation dose of digital tomosynthesis was a little higher than that of digital radiography, which was evidently lower than that of the high- and low-dose MDCT. Digital tomosynthesis had the lowest costeffectiveness rate, while MDCT-2D-Mip had the highest among the four examinations. In the current research, our result revealed that digital tomosynthesis possessed the same localization capacity as MDCT-2D-MIP. The report on the digital tomosynthesis showed that it would be cost-effective when 0.6 MDCT examinations were transferred to the digital tomosynthesis on a weekly basis. ${ }^{29}$ Thus, the costeffectiveness seems to be guaranteed.

The data from this study suggested that the capacity of digital tomosynthesis in diagnosing urinary stones was as good as MDCT with lower mean ESD and cost-effectiveness. It might be a substitute for digital radiography and the preferred technology to diagnose urinary stones with higher sensitivity. Furthermore, digital tomosynthesis owned a similar localization function to KUB, ultrasound, and MDCT. Thus, this technique might be helpful to improve or 
substitute KUB and ultrasound in patients with urinary stones. However, the MDCT might still be the standard in detecting urinary calculus with a high sensitivity even after stone passage for a long time, because it can directly show stone or visualize the secondary MDCT signals of the obstructions of urinary, including ureterectasis and periureteral fat strangulation. ${ }^{11,12,15,18}$

There were still many limitations in this study. First, the sample size was small. Further studies with a larger sample are essential for clarifying the importance of digital tomography in the diagnosis and follow-up of urinary calculi. Second, digital tomosynthesis showed 83 stones, and all of those stones were detected by CT in this study, implying that there were no falsenegative samples of digital tomosynthesis. However, the falsenegative samples of digital tomosynthesis have not been determined in our study. Third, it would be better to measure both the entrance dose and the ESD at the same time. In this study, as we did not have the conditions to test entrance dose yet, we only measured the ESD.

In summary, the diagnostic value of application of digital tomosynthesis was as good as MDCT. What's more, its ESD and cost-effectiveness rate were both lower than those of MDCT. Hence, digital tomosynthesis might be useful in the diagnosis and follow-up of urinary stones.

\section{Authors' Contributions}

S.L. and W.F. conceived and designed the experiments; S.L., P.N., and H.W. performed the experiments; P.N., J.G., and Q.S. analyzed the data; W.X. and W.F. contributed reagents/materials/analysis tools: S.L. and P.N. wrote the article.

\section{Author Disclosure Statement}

No competing financial interests exist.

\section{Funding Information}

No funding was received for this article.

\section{References}

1. Turney BW, Reynard JM, Noble JG, Keoghane SR. Trends in urological stone disease. BJU Int 2012;109:1082-1087.

2. Levine JA, Neitlich J, Verga M, Dalrymple N, Smith RC. Ureteral calculi in patients with flank pain: Correlation of plain radiography with unenhanced helical CT. Radiology 1997;204:27-31.

3. Roth CS, Bowyer BA, Berquist TH. Utility of the plain abdominal radiograph for diagnosing ureteral calculi. Ann Emerg Med 1985;14:311-315.

4. Mutgi A, Williams JW, Nettleman M. Renal colic. Utility of the plain abdominal roentgenogram. Arch Intern Med 1991;151:1589-1592.

5. Rosen CL, Brown DF, Sagarin MJ, Chang Y, McCabe CJ, Wolfe RE. Ultrasonography by emergency physicians in patients with suspected ureteral colic. J Emerg Med 1998; 16:865-870.

6. Henderson SO, Hoffner RJ, Aragona JL, Groth DE, Esekogwu VI, Chan D. Bedside emergency department ultrasonography plus radiography of the kidneys, ureters, and bladder vs intravenous pyelography in the evaluation of suspected ureteral colic. Acad Emerg Med 1998;5:666671.

7. Fowler KA, Locken JA, Duchesne JH, Williamson MR. US for detecting renal calculi with nonenhanced CT as a reference standard. Radiology 2002;222:109-113.

8. Ulusan S, Koc Z, Tokmak N. Accuracy of sonography for detecting renal stone: Comparison with CT. J Clin Ultrasound 2007;35:256-261.

9. Sinclair D, Wilson S, Toi A, Greenspan L. The evaluation of suspected renal colic: Ultrasound scan versus excretory urography. Ann Emerg Med 1989;18:556-559.

10. Pfister SA, Deckart A, Laschke S, et al. Unenhanced helical computed tomography vs intravenous urography in patients with acute flank pain: Accuracy and economic impact in a randomized prospective trial. Eur Radiol 2003;13:25132520.

11. Shine S. Urinary calculus: IVU vs. CT renal stone? A critically appraised topic. Abdom Imaging 2008;33:41-43.

12. Chu G, Rosenfield AT, Anderson K, Scout L, Smith RC. Sensitivity and value of digital CT scout radiography for detecting ureteral stones in patients with ureterolithiasis diagnosed on unenhanced CT. AJR Am J Roentgenol 1999; 173:417-423.

13. Remer EM, Herts BR, Streem SB, Hesselink DP, Shiesly DA, Yost AJ, Baker ME. Spiral noncontrast CT versus combined plain radiography and renal US after extracorporeal shock wave lithotripsy: Cost-identification analysis. Radiology 1997;204:33-37.

14. Sheafor DH, Hertzberg BS, Freed KS, Carroll BA, Keogan MT, Paulson EK, DeLong DM, Nelson RC. Nonenhanced helical CT and US in the emergency evaluation of patients with renal colic: Prospective comparison. Radiology 2000; 217:792-797.

15. Smith RC, Rosenfield AT, Choe KA, Essenmacher KR, Verga M, Glickman MG, Lange RC. Acute flank pain: Comparison of non-contrast-enhanced CT and intravenous urography. Radiology 1995;194:789-794.

16. Zagoria RJ. Retrospective view of "diagnosis of acute flank pain: value of unenhanced helical CT." AJR Am J Roentgenol 2006;187:603-604.

17. Lumerman J, Gershbaum MD, Hines J, Nardi P, Beuchert P, Katz DS. Unenhanced helical computed tomography for the evaluation of suspected renal colic in the adolescent population: A pilot study. Urology 2001;57:342-346.

18. Tack D, Sourtzis S, Delpierre I, de Maertelaer V, Gevenois PA. Low-dose unenhanced multidetector CT of patients with suspected renal colic. AJR Am J Roentgenol 2003; 180:305-311.

19. Kluner C, Hein PA, Gralla O, Hein E, Hamm B, Romano V, Rogalla P. Does ultra-low-dose CT with a radiation dose equivalent to that of KUB suffice to detect renal and ureteral calculi? J Comput Assist Tomogr 2006;30:44-50.

20. Poletti PA, Platon A, Rutschmann OT, Schmidlin FR, Iselin $\mathrm{CE}$, Becker CD. Low-dose versus standard-dose CT protocol in patients with clinically suspected renal colic. AJR Am J Roentgenol 2007;188:927-933.

21. Liu W, Esler SJ, Kenny BJ, Goh RH, Rainbow AJ, Stevenson GW. Low-dose nonenhanced helical CT of renal colic: Assessment of ureteric stone detection and measurement of effective dose equivalent. Radiology 2000;215: $51-54$.

22. Badea C, Kolitsi Z, Pallikarakis N. A 3D imaging system for dental imaging based on digital tomosynthesis and cone beam CT. Proceedings of Conference of the International 
Federation for Medical and Biological Engineering 2001;2: 739-741.

23. Stiel GM, Stiel LG, Klotz E, Nienaber CA. Digital flashing tomosynthesis: A promising technique for angiocardiographic screening. IEEE Trans Med Imaging 1993;12:314 321.

24. Warp RJ, Godfrey DJ, Dobbins JT, 3rd. Applications of matrix inverse tomosynthesis. Proc SPIE Med Imaging 2000;3977:376-383.

25. Duryea J, Dobbins JT, 3rd, Lynch JA. Digital tomosynthesis of hand joints for arthritis assessment. Med Phys 2003; 30:325-333.

26. Some $\mathrm{S}$, Kasuga $\mathrm{T}$, Sakai $\mathrm{F}$, et al. Image processing in the digital tomosynthesis for pulmonary imaging. Eur Radiol 1995;5:96-101.

27. Niklason LT, Christian BT, Niklason LE, et al. Digital tomosynthesis in breast imaging. Radiology 1997;205:399406.

28. Mermuys K, De Geeter F, Bacher K, Van De Moortele K, Coenegrachts K, Steyaert L, Casselman JW. Digital tomosynthesis in the detection of urolithiasis: Diagnostic performance and dosimetry compared with digital radiography with MDCT as the reference standard. AJR Am J Roentgenol 2010;195:161-167.

29. Purchasing CFE-B. Evaluation Report: Tomosynthesis for General Radiography. London, UK: NHS Purchasing and Supply Agency, 2009.
Address correspondence to: Weihua Feng, PhD

Department of Radiology The Affiliated Hospital of Qingdao University

No. 16 Jiangsu Road

Qingdao 266000

Shinan District

China

E-mail:13808995736@163.com

$\quad$ Abbreviations Used
$2 \mathrm{D}=$ two dimensional
2D-MIP $=$ two-dimensional-maximum intensity
projection
$3 \mathrm{D}=$ three dimensional
$\mathrm{CEA}=$ cost-effectiveness analysis
$\mathrm{CT}=$ computed tomography
$\mathrm{ESD}=$ entrance surface dose
$\mathrm{IVP}=$ intravenous pyelography
$\mathrm{KUB}=$ kidney, ureter, and bladder radiography
$\mathrm{MDCT}=$ multidetector computed tomography
$\mathrm{PACS} / \mathrm{HIS}=$ picture archiving and communication systems
$\quad$ and hospital information system
$\mathrm{US}=$ ultrasonography

